\title{
The effect of probe inaccuracies on the quantitative model-based analysis of high angle annular dark field scanning transmission electron microscopy images
}

\author{
G.T. Martinez ${ }^{\mathrm{a}, *}$, A. De Backer ${ }^{\mathrm{a}}$, A. Rosenauer ${ }^{\mathrm{b}}$, J. Verbeeck ${ }^{\mathrm{a}}$, S. Van Aert ${ }^{\mathrm{a}}$ \\ a Electron Microscopy for Materials Science (EMAT), University of Antwerp, Gronenborgerlaan 171, 2020 Antwerp, Belgium \\ ${ }^{\mathrm{b}}$ Institut für Festkörperphysik, Universität Bremen, Otto-Hahn-Alle 1, D-28359 Bremen, Germany
}

\section{A R T I C L E I N F O}

\section{Article history:}

Received 20 September 2013

Received in revised form

13 December 2013

Accepted 15 December 2013

Available online 27 December 2013

\section{Keywords:}

HAADF STEM

Probe aberrations

Statistical parameter estimation theory

STEM simulations

\begin{abstract}
A B S T R A C T
Quantitative structural and chemical information can be obtained from high angle annular dark field scanning transmission electron microscopy (HAADF STEM) images when using statistical parameter estimation theory. In this approach, we assume an empirical parameterized imaging model for which the total scattered intensities of the atomic columns are estimated. These intensities can be related to the material structure or composition. Since the experimental probe profile is assumed to be known in the description of the imaging model, we will explore how the uncertainties in the probe profile affect the estimation of the total scattered intensities. Using multislice image simulations, we analyze this effect for $C s$ corrected and non-Cs corrected microscopes as a function of inaccuracies in cylindrically symmetric aberrations, such as defocus and spherical aberration of third and fifth order, and non-cylindrically symmetric aberrations, such as 2-fold and 3-fold astigmatism and coma.
\end{abstract}

(c) 2013 Elsevier Ltd. All rights reserved.

\section{Introduction}

The use of a high angle annular dark field detector in a scanning transmission electron microscope (HAADF STEM) allows one to obtain images whose contrast is sensitive to structural and chemical information of the material under study. The intensities of these images scale with the mean atomic number $Z$ of the atomic columns, hence the name $Z$-contrast imaging (Pennycook and Jesson, 1991). It has also been demonstrated that the intensities can be related to the number of atoms present in each atomic column (Van Aert et al., 2011, 2013; LeBeau et al., 2010; De Backer et al., 2013). Therefore, this technique is widely used for chemical and structural analyses of materials at the atomic level. To analyze HAADF STEM images as accurately and precisely as possible, quantitative methods are needed. In order to analyze HAADF STEM images quantitatively, several approaches have been proposed (Rosenauer et al., 2009; Robb et al., 2012; LeBeau et al., 2008; Kotaka, 2010). Furthermore, statistical parameter estimation theory has been introduced as an alternative method to extract quantitative information from HAADF STEM images, such as chemical composition (Van Aert et al., 2009; Martinez et al., 2014) or number of atoms (Van Aert et al., 2011, 2013; De Backer et al., 2013),

\footnotetext{
* Corresponding author. Tel.: +32 (0) 32653317.

E-mail address: gerardo.martinez@uantwerpen.be (G.T. Martinez).
}

with high accuracy and precision. In this framework, HAADF STEM images are described using a simplified parameterized empirical imaging model. The unknown parameters of this model are then estimated in an iterative way by fitting this model to the experimental images using a criterion of goodness of fit, such as least squares, least absolute squares or maximum likelihood (den Dekker et al., 2005; Van Aert et al., 2005). In this manner, the total intensity of scattered electrons can be quantified atomic column-by-atomic column, which is particularly interesting due to its sensitivity for the chemical composition. The use of this methodology has been shown to allow for a chemical quantification of interfaces (Van Aert et al., 2009), and to study the structure and composition of nanoparticles (Bals et al., 2011) and nanoclusters (Bals et al., 2012). The research on nanostructured materials such as nanoparticles is of great interest because of their wide applications in different fields, such as catalysts for example (Yu et al., 2012). Model-based quantification of HAADF STEM images has been presented in Van Aert et al. (2009, 2012) and an extensive analysis on the inherent limitations of this methodology as a tool for atom counting has been explained in De Backer et al. (2013). Furthermore, the model assumptions and validity for single atomic column chemical quantification have been discussed in Martinez et al. (2014). In this work, we analyze how inaccuracies in the probe aberrations, which are usually assumed to be known, affect the estimation of the scattered intensities of the atomic columns. For that purpose, we will make use of multislice simulations under the absorptive potential 
approach (Ishizuka, 2002) because of their suitability to describe electron-sample interactions for thin samples. In Section 2, we will review the model-based analysis for quantification of HAADF STEM images. In Section 3 we will describe the simulation methodology and settings. We consider the example of Pt as a test material because of its increasing interest in the catalyst research community (Chen and Holt-Hindle, 2010). However, the analysis can be extended to all types of materials. In Section 4, the results will be presented and discussed. Finally, in Section 5, conclusions are drawn.

\section{Model-based parameter estimation}

Model-based electron microscopy has recently been reviewed in Van Aert et al. (2012), where a wide scope of applications is discussed as well. For the particular case of quantification of $Z$ contrast HAADF STEM images, the methodology is presented in Van Aert et al. (2009). Quantitative information is then obtained by measuring total scattered atom column intensities using statistical parameter estimation theory. An empirical incoherent imaging model is used to measure these quantities. This parametric model describes the expectations of the pixel values of the HAADF STEM image. If we assume an incoherent model for this purpose, we can describe the electron-sample interaction as the convolution between an object function and the probe intensity (Pennycook and Jesson, 1991; Nellist, 2007):

$f_{k l}(\boldsymbol{\theta})=f\left(\mathbf{r}_{k, l} ; \boldsymbol{\theta}\right)=\mathrm{O}\left(\mathbf{r}_{k, l} ; \boldsymbol{\theta}\right) * \mathrm{P}\left(\mathbf{r}_{k, l}\right)$

where $\mathrm{O}(\mathbf{r} ; \boldsymbol{\theta})$ is the object function depending on the unknown structure parameters $\boldsymbol{\theta}$ and $\mathrm{P}(\mathbf{r})$ is the probe function depending on a set of probe parameters including acceleration voltage, defocus, aperture semi-angle, spherical aberration constant and high order aberration coefficients. The indices $(k, l)$ correspond to the STEM probe at position $\mathbf{r}_{k, l}=\left(x_{k} y_{l}\right)^{T}$.

The information about the sample and the HAADF detector is incorporated in the object function, which describes the scattering interaction of the probe with the projected potential recorded at the detector plane. This function is sharply peaked at the atom column positions and can be defined as a superposition of Gaussian peaks:

$\mathrm{O}\left(\mathbf{r}_{k, l} ; \boldsymbol{\theta}\right)=\zeta+\sum_{n=1}^{N} \eta_{n} \exp \left(\frac{-\left(x_{k}-\beta_{x_{n}}\right)^{2}-\left(y_{l}-\beta_{y_{n}}\right)^{2}}{2 \rho^{2}}\right)$

where $\zeta$ is a constant background, $N$ is the total number of atomic columns to be analyzed, $\rho$ is the width of a Gaussian peak, $\eta_{n}$ is the height of the $n$th Gaussian peak, $\beta_{x_{n}}$ and $\beta_{y_{n}}$ are the $x$ - and $y$-coordinates of the $n$th atomic column, respectively.

Thus, the unknown parameters are given by the parameter vector:

$\boldsymbol{\theta}=\left(\beta_{x_{1}}, \ldots, \beta_{x_{N}}, \beta_{y_{1}}, \ldots, \beta_{y_{N}}, \rho, \eta_{1}, \ldots, \eta_{N}, \zeta\right)^{T}$

In order to estimate the unknown parameters, use is made of the uniformly weighted least squares estimator. The function parameters are then estimated by minimizing the least squares sum using an iterative routine. After the estimation of the unknown parameters from the experimental images, the volumes under the Gaussian peaks above the background are used as a sensitive measure to extract quantitative information. It has been shown that this measure is proportional to the total intensity of electrons scattered by a specific atomic column that was integrated at the HAADF detector (Van Aert et al., 2009). These volumes can be computed as follows:

$\mathrm{V}_{n}=2 \pi \eta_{n} \rho^{2}$
The function $\mathrm{P}(\mathbf{r})$ in Eq. (1) is the STEM probe that scans over the sample. It is given by the following expression:

$\mathrm{P}(\mathbf{r})=|p(\mathbf{r})|^{2} * S(\mathbf{r})$

where $|p(\mathbf{r})|^{2}$ is the coherent point source contribution and $S(\mathbf{r})$ represents the incoherent source size effects (Born and Wolf, 1998). The STEM probe formation takes place at the objective lens, which strongly focuses the electron beam to form a crossover which is described by the function $p(\mathbf{r})$. This function is given by the inverse Fourier transform of the transfer function of the objective lens $T(\mathbf{g})$, which is defined as:

$T(\mathbf{g})=A(\mathbf{g}) \exp (i \chi(\mathbf{g}))$

with $A(\mathbf{g})$, the so-called aperture function, being a circular top-hat function with unity height and radius $g_{a p}$. The objective aperture semi-angle $\alpha_{0}$ is related to this function by the equality $\alpha_{0}=g_{a p} \lambda$, where $\lambda$ is the electron wavelength. The exponential term in Eq. (6) describes a phase shift $\chi(\mathbf{g})$ due to the objective lens aberrations. The function $\chi(\mathbf{g})$ incorporates the effect of rotationally symmetric aberrations, such as defocus and spherical aberration of third and fifth order. Non-symmetric aberrations such as astigmatism and coma can also be included in this function for a more accurate description of the probe profile (Haider et al., 2000). Extensive work has been performed in order to measure the objective lens aberrations (Haider et al., 2000; Wong et al., 1992; Uhlemannm and Haider, 1998; Batson, 2006; Krivanek et al., 2008). The most recent aberration corrected instruments incorporate automated routines to measure the residual aberrations on a daily basis. Computer assisted routines have been developed to analyze diffractogram tableaus, so-called Zemlin-tableaus (Zemlin et al., 1978), and to address residual aberrations and their stability during the experiment (Barthel and Thust, 2010). For STEM, use is made of far-field shadow images, so-called Ronchigrams, to perform this task (Lupini et al., 2010). The parametric model proposed in Van Aert et al. (2009) assumes the probe function to be known. This probe function is determined by the instrument. The characteristic probe aberrations should be measured experimentally. Residual aberrations can be measured with different methodologies up to the unavoidable experimental limitations, including reading noise, fluctuations of the probe current due to microscope instabilities, hardware and software computational restrictions (Barthel and Thust, 2010). Thus, there is an unavoidable uncertainty in the probe profile. Therefore, we will study how these inaccuracies affect the parameter estimates, the scattered intensities given by Eq. (4), in particular. We will show our analysis as a function of inaccuracies in defocus $\left(C_{1}\right)$, spherical aberration of third $\left(C_{3}\right)$ and fifth order $\left(C_{5}\right), 2$-fold $\left(A_{1}\right)$ and 3-fold $\left(A_{2}\right)$ astigmatism and coma $\left(B_{2}\right)$.

\section{HAADF STEM simulation study}

The analysis presented in this work will make use of multislice simulations under the absorptive potential approximation (Ishizuka, 2002) using the StemSim software (Rosenauer and Schowalter, 2007). The absorptive potential approximation is computationally less demanding while it still describes the image intensities properly up to $50 \mathrm{~nm}$ thickness of the sample (Rosenauer et al., 2009). We simulated a Pt structure in [100] and [1 10 ] zone axis up to 75 atoms thickness, that is, $\approx 30 \mathrm{~nm}$ and $\approx 21 \mathrm{~nm}$, respectively. The simulated images were convolved with a Gaussian function to account for spatial incoherence. We consider two cases: a Cs corrected and a non-Cs corrected microscope under their corresponding Scherzer conditions. The simulation settings are summarized in Table 1.

Using the theory described in Section 2, the scattered intensities, given by Eq. (4), have been estimated from the simulated images 


\section{Cs corrected}
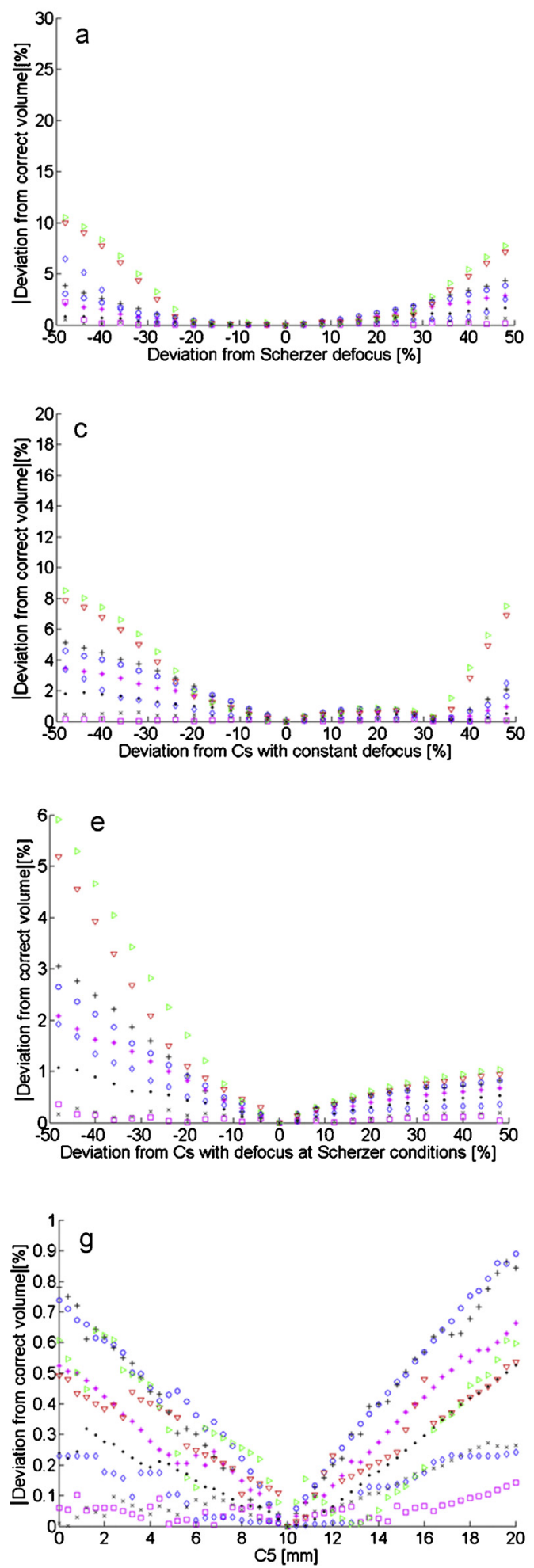

Non-Cs corrected
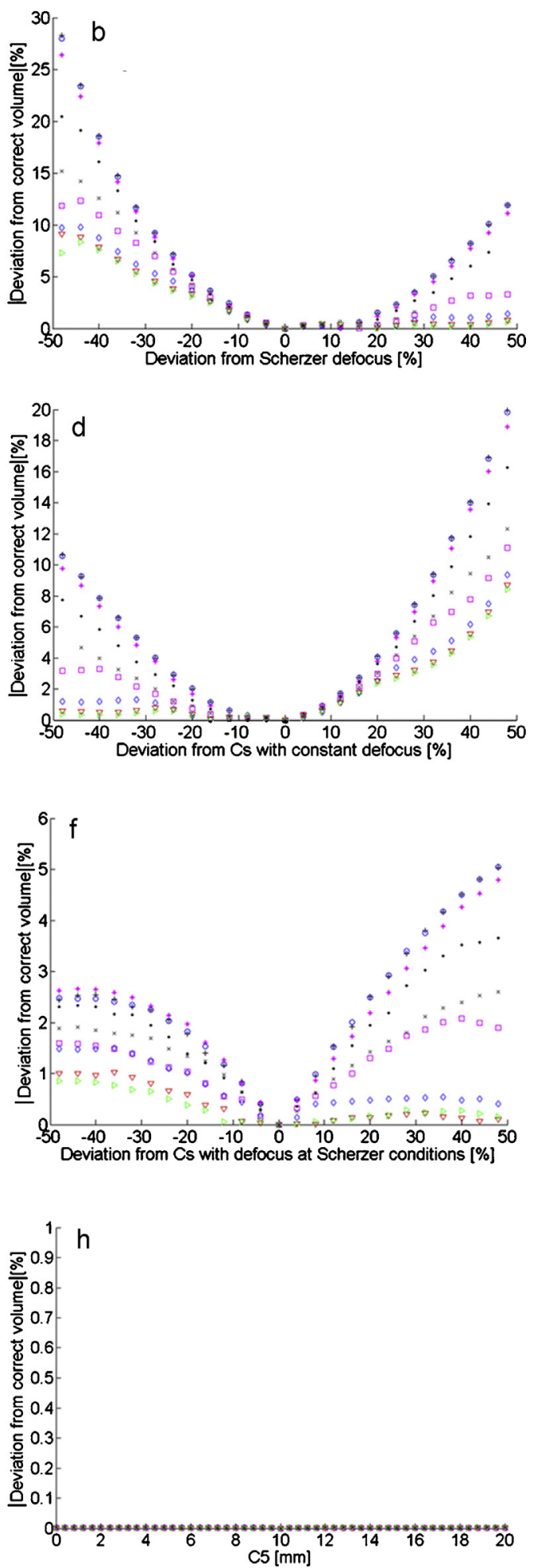

1 atom $\nabla 5$ atoms $\quad 10$ atoms $\square 15$ atoms $\times 20$ atoms

$\cdot 30$ atoms +45 atoms $\circ 60$ atoms +75 atoms

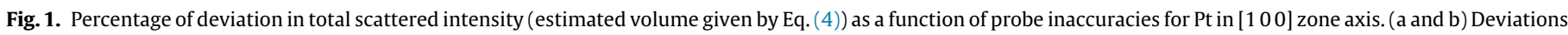

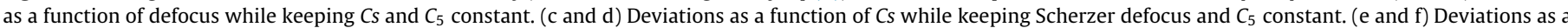
function of $C s$ while adapting defocus to meet Scherzer conditions. $C_{5}$ is kept constant. ( $\mathrm{g}$ and $\mathrm{h}$ ) Deviations as a function of $C_{5}$ while keeping defocus and $C_{s}$ constant. 
Cs corrected
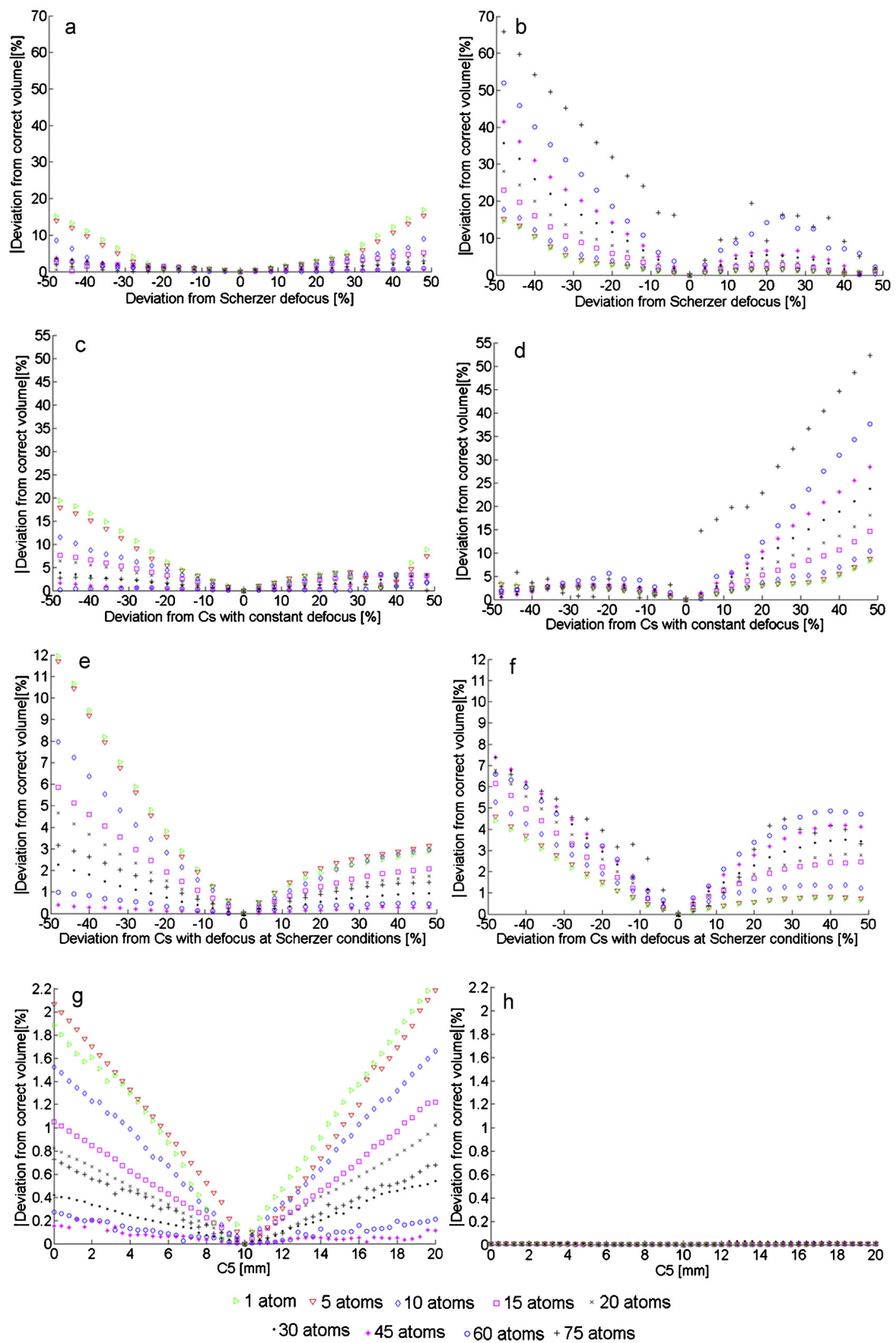

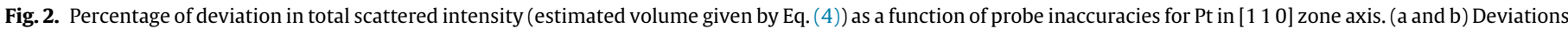

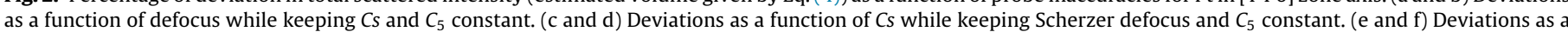
function of $C s$ while adapting defocus to meet Scherzer conditions. $C_{5}$ is kept constant. $\left(\mathrm{g}\right.$ and $\mathrm{h}$ ) Deviations as a function of $C_{5}$ while keeping defocus and $C_{5}$ constant. 


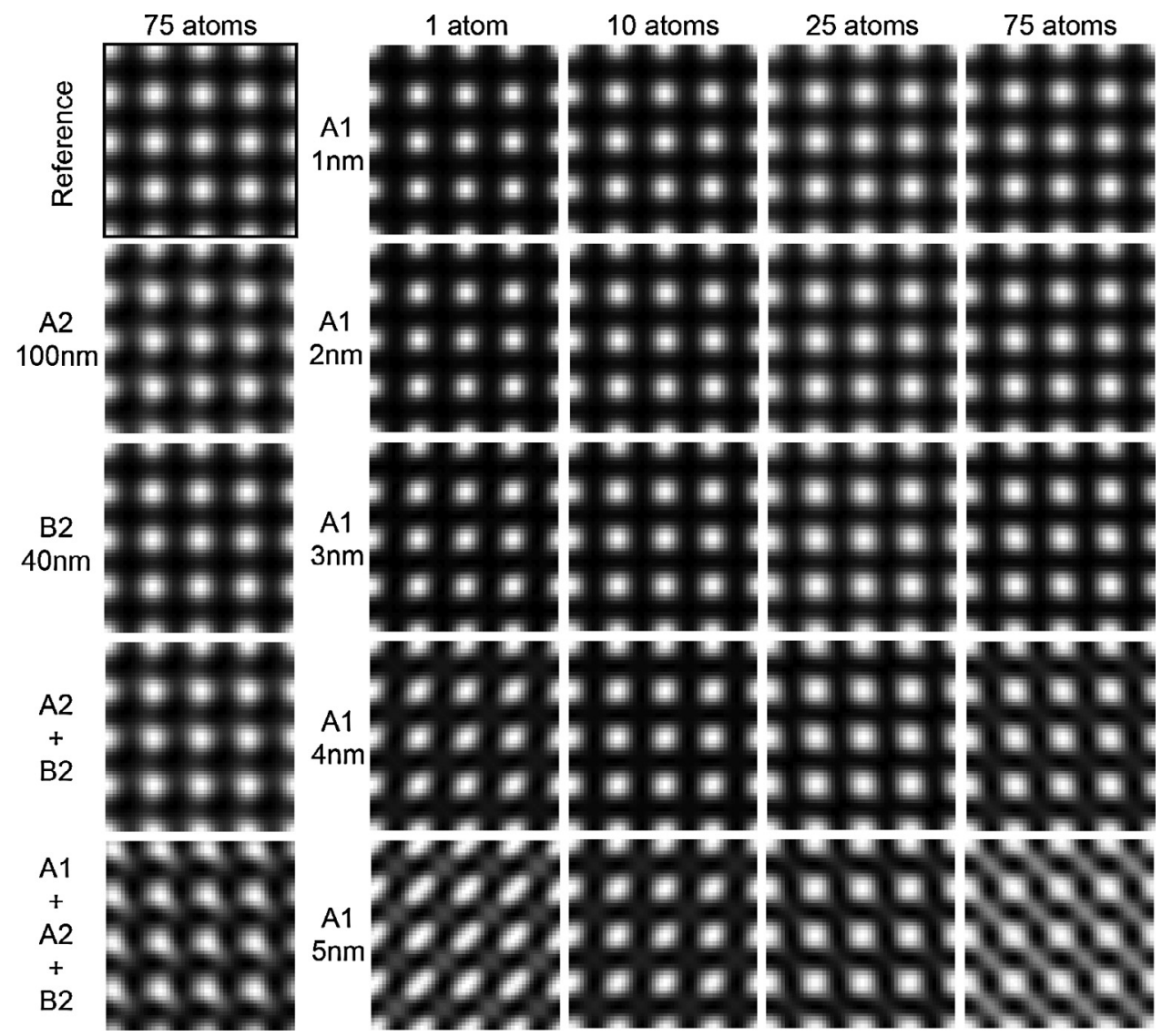

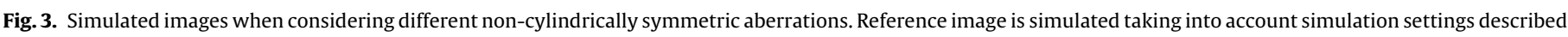

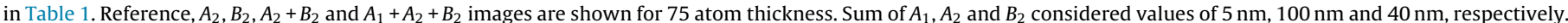

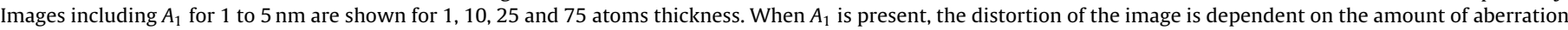

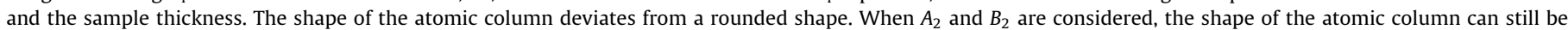
modeled by rounded Gaussians. The effect of $A_{1}$ is predominant in respect to $A_{2}$ and $B_{2}$.

assuming variation in the probe aberrations around the true aberrations. As presented in Martinez et al. (2014), fitting a constant background to simulations is not needed, since this background considers the signals that do not originate from the sample under study, such as the sample support or the grid. Consequently, a background with value 0 was considered for this analysis. Furthermore, we will study the effect on the scattered intensities for $1,5,10,15$, $20,30,45,60$ and 75 atoms thickness. The analysis of simulated

Table 1

Settings used for multislice simulations.

\begin{tabular}{ll} 
Microscope & Cs corrected/non-Cs corrected \\
Acceleration voltage HT & $300 \mathrm{kV}$ \\
Defocus $C_{1}$ & $-8.8741 \mathrm{~nm} /-48.606 \mathrm{~nm}$ \\
Spherical aberration $C_{3}$ & $0.04 \mathrm{~mm} / 1.2 \mathrm{~mm}$ \\
Spherical aberration $C_{5}$ & $10 \mathrm{~mm}$ \\
Objective aperture angle $\alpha$ & $21.1 \mathrm{mrad} / 9 \mathrm{mrad}$ \\
Spatial incoherence of source FWHM & $0.7 \AA / 1.23 \AA$ \\
Number of unit cells per supercell & $9 \times 9$ \\
HAADF inner collection angle & $60 \mathrm{mrad}$ \\
HAADF outer collection angle & $190 \mathrm{mrad}$ \\
Maximum specimen thickness [1 00] & $29.4 \mathrm{~nm}$ \\
Maximum specimen thickness [110] & $20.8 \mathrm{~nm}$ \\
Debye-Waller factor B & $0.384 \AA$ \\
Pixel size [1 0 0] & $0.1569 \AA$ \\
Pixel size [1 10] & $0.1121 \AA$ \\
Non-cylindrically symmetric aberrations: & \\
2-Fold astigmatism $\left(A_{1}\right)$ & $1-5 \mathrm{~nm}$ at $\pi / 4$ \\
3-Fold astigmatism $\left(A_{2}\right)$ & $100 \mathrm{~nm}$ at $\pi / 4$ \\
Coma $\left(B_{2}\right)$ & $40 \mathrm{~nm}$ at $\pi / 4$ \\
\hline
\end{tabular}

images for two different zone axes is intended to consider different atomic column spacings, having a different effect on the cross-talk of the electron beam through the sample. Typical values for the non-cylindrically symmetric aberrations have been considered in the image simulations resulting from computer assisted alignment of the probe corrector in a FEI Titan cubed $80-300 \mathrm{kV}$. Regularly, values of 2-fold astigmatism $\left(A_{1}\right)$ below $5 \mathrm{~nm}$ are obtained. For 3fold $\left(A_{2}\right)$ astigmatism and coma $\left(B_{2}\right)$, the amount of aberration used is $100 \mathrm{~nm}$ and $40 \mathrm{~nm}$, respectively. These aberrations considered a rotation angle of $\pi / 4$.

\section{Results and discussion}

\subsection{Analysis of cylindrically symmetric aberrations}

The analysis for cylindrically symmetric aberrations is presented in Figs. 1 and 2, where the effect of variations around the true aberration coefficients on the total scattered column intensities is shown. Figs. 1 and 2(a and b) show the deviations of total scattered intensity in percentage with respect to variation of defocus from Scherzer defocus conditions for a Cs corrected and non-CS corrected microscope, respectively. Negative and positive deviations are considered corresponding to underfocus and overfocus, respectively. All other microscope settings are given in Table 1. For the $C s$ corrected instrument, it follows that a variation in defocus of $\pm 20 \%$ results in a deviation of $\approx 1 \%$ regardless the number of atoms present in the sample or zone axis. For larger variations, the deviation increases significantly and depends on the number of atoms, 
resulting in a higher deviation when few atoms are present. In the non-Cs corrected example, the effect of the variation in defocus is more pronounced and highly dependent on atomic column spacing, that is, the chosen zone axis. For the [ 1000$]$ zone axis, the deviation reaches $\approx 5 \%$ for a variation of $\pm 20 \%$. For [ 1110 ] zone axis, the same amount of variation in defocus leads to a deviation up to $\approx 30 \%$ for large number of atoms. Figs. 1 and 2(c and d) show the deviation of the total scattered intensity as a function of variations in $C s$ while keeping the defocus value constant. In this case, a $\pm 20 \%$ variation around the true $C s$ value leads to a deviation of $\approx 2 \%$ in [ 100 ] zone axis and $\approx 5 \%$ in [ 1110$]$ ] for the Cs corrected microscope. Higher deviations are found for the non-Cs corrected example when a larger number of atoms is present. In comparison with Figs. 1 and 2(e and f), where the defocus is adapted to meet Scherzer conditions with varying $C s$, the deviation in total scattered intensities is significantly smaller. The deviation is less than $\approx 4 \%$ in the $\pm 20 \%$ defocus variation range regardless of number of atoms, zone axis or Cs correction. Finally, Figs. 1 and $2(\mathrm{~g}$ and $\mathrm{h}$ ) show the effect of varying $C_{5}$. These results indicate very small deviations in total scattered intensities for a range of $\pm 10 \mathrm{~mm}$ around the true $C_{5}$. However, deviations are larger for the $C s$ corrected microscope as compared to the non-Cs corrected microscope. Modification of the probe profile due to this aberration is indeed more prominent in case of $C s$ correction. For the case of the non-Cs corrected microscope, the effect is practically none regardless of zone axis, due to the predominant effect of $C s$ aberration on the probe profile. An overall observation of this study is that Cs correction is more sensitive to inaccuracies in the probe settings for a few number of atoms. Estimated scattered intensities obtained from images of a nonCs corrected microscope are clearly affected with respect to the number of atoms present in the crystal and atomic column spacing. However, deviations in estimated scattered intensities $\approx 1$ to $5 \%$ are observed when the experimental uncertainties in the true probe settings are in the $\pm 20 \%$ range for most cases. The quantification of scattered intensities is less affected when the probe profile is significantly peaked. This demonstrates a robust behavior of this model-based method with respect to probe inaccuracies for cylindrically symmetric aberrations.

\subsection{Analysis of non-cylindrically symmetric aberrations}

As explained in Section 2, the proposed model considers only cylindrically symmetric aberrations, such as defocus and spherical aberrations of third $(C s)$ and fifth $\left(C_{5}\right)$ order. However, in $C s$ corrected microscopes, the remaining non-cylindrically symmetric aberrations, such as 2 -fold $\left(A_{1}\right), 3$-fold $\left(A_{2}\right)$ astigmatism and coma $\left(B_{2}\right)$, can have an important effect on the image, which is seldomly observed in non-Cs corrected instruments, because of the predominant effect of $C s$. Therefore, we analyze how these aberrations affect estimated scattered intensities when taking images with a $C s$ corrected microscope. We compare the scattered intensities obtained at Scherzer conditions using the simulations settings from Table 1 for the $\left[\begin{array}{ll}10 & 0\end{array}\right]$ zone axis. Maximum values of 2-fold, 3-fold astigmatism and coma corresponding to typical values obtained when using computer assisted software to align the probe corrector in a Titan cubed $80-300 \mathrm{kV}$ are considered for the simulations. Usually, values of $A_{1}$ should be below $5 \mathrm{~nm}$ when the corrector is properly aligned. This aberration is corrected at the moment of recording the image and it may even slightly vary during the acquisition. Consequently, we simulated a series of images including this aberration in $1 \mathrm{~nm}$ steps, ranging from 1 to $5 \mathrm{~nm}$. The simulated images are shown in Fig. 3. Deviations from a rounded shape of the atomic column are observed depending on the amount of 2 -fold astigmatism, but also depending on the number of atoms present. Fig. 4(a) shows the deviation from the estimated scattered intensity with respect to number of atoms for the different values of $A_{1}$. When comparing
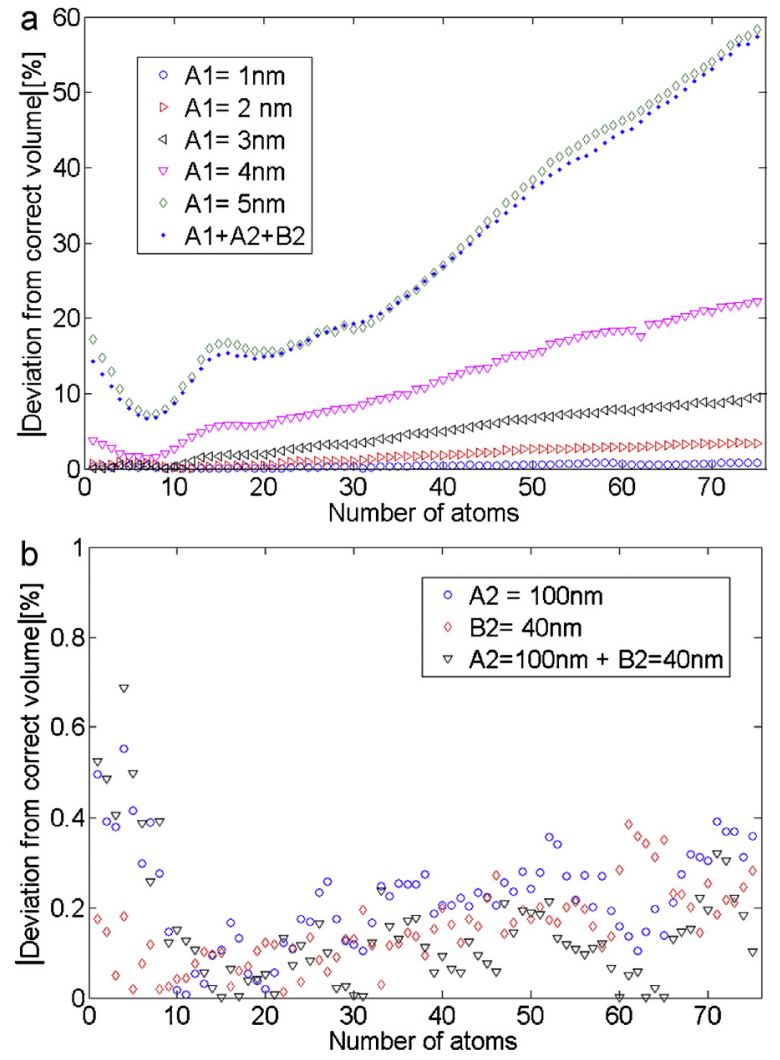

Fig. 4. Percentage of deviation in total scattered intensity (estimated volume given by Eq. (4)) as a function of number of atoms. (a) Deviation from correct volume when 2-fold $A_{1}$ astigmatism is included for $1-5 \mathrm{~nm}$. Sum of $A_{1}, A_{2}$ and $B_{2}$ for $5 \mathrm{~nm}$, $100 \mathrm{~nm}$ and $40 \mathrm{~nm}$, respectively, is also shown. There is a predominant effect of $A_{1}$. (b) Deviation from correct volume when 3-fold $A_{2}$ astigmatism and coma $B_{2}$, is considered for $100 \mathrm{~nm}$ and $40 \mathrm{~nm}$, respectively.

these results with the images shown in Fig. 3, it can be concluded that the deviation with respect to the reference case remains very small as long as the column shape is visually round. For values of $A_{1}$ up to $3 \mathrm{~nm}$, the fitting procedure is able to retrieve most of the atomic column intensity even when considering a Gaussian shape to model the column images. In contrast, for $5 \mathrm{~nm} 2$-fold astigmatism, deviations are large indicating the need to correct for this aberration manually after using computer assisted software. Special attention is drawn to the case of 10 atoms thickness showing a minimum in the deviation. In Fig. 3, the nearly rounded shape of the atomic column is observable at this thickness, corresponding to the turning point of the asymmetry of the column. For values below 10 atoms, the asymmetry direction is observed indeed from lower left to upper right of the image, whereas for larger number of atoms, the asymmetry direction changes from lower right to upper left of the image. This observation is expected due to the asymmetry of the probe profile while propagating inside the crystal. Fig. 3 also shows simulated images when $A_{2}$ and $B_{2}$ are included for 75 atoms thickness. For these aberrations, the deviation of a rounded shape is less prominent at the maximum values usually obtained after software correction, that is at $100 \mathrm{~nm}$ and $40 \mathrm{~nm}$, respectively. Fig. 4(b) shows that the deviation from the reference scattered intensity when considering these aberrations separately or combined is less than $1 \%$. Finally, a simulated image considering the maximum values $(5 \mathrm{~nm}$ $-100 \mathrm{~nm}-40 \mathrm{~nm}$ ) for the three aberrations $A_{1}, A_{2}$ and $B_{2}$ together is shown in Fig. 3 for 75 atoms thickness. The combined aberrations result in a clearly distorted image. It is found that the effect of $A_{1}$ predominates and that the deviation with respect to the scattered intensity reference is very similar to the curve for $5 \mathrm{~nm} 2$-fold 
astigmatism in Fig. 4. In conclusion, the parametric model shows a robust behavior for non-cylindrically symmetric aberrations even if they are not taken into account in the probe profile.

\section{Conclusions}

Statistical parameter estimation theory has been shown to be a reliable technique to extract quantitative structural and chemical information from HAADF STEM images. This approach relies on estimating unknown structure parameters of an empirical parameterized imaging model. From these estimated parameters, the total scattered intensity of the atomic columns can be determined. The total scattered intensity is then related to structure or composition of the atomic column. In this approach, it is generally assumed that the scanning probe profile is known. In this work, we have explored how experimental uncertainties of the probe affect the estimated total scattered intensity. Using multislice simulations under Scherzer conditions, we have analyzed this effect for a CS and non-Cs corrected microscope as a function of uncertainties in cylindrically symmetric aberrations, such as defocus and spherical aberration of third and fifth order for two different atomic column spacings. In general, it can be concluded that deviations in scattered intensities are between $\approx 1$ and $5 \%$ when the experimental uncertainties are in the $\pm 20 \%$ range for most cases. Reduced atomic column spacing and increasing number of atoms may have an important effect for non-Cs corrected instruments, whereas the scattered intensities for a Cs corrected instrument are more affected for few atoms present in the sample. Moreover, when adapting defocus for a varying $C s$ in order to keep Scherzer conditions, the deviations are clearly reduced. The effect of $C_{5}$ can practically be neglected for a non-Cs corrected microscope and has very little influence for the Cs corrected case. Since the model only considers cylindrically symmetric aberrations while estimating the model parameters, we analyzed how non-cylindrically symmetric aberrations, such as 2-fold and 3fold astigmatism and coma, affect the estimation of the scattered intensities for a Cs corrected instrument, since for a non-Cs corrected microscope, the effect of these aberrations is overruled by the rounded distortion caused by Cs. It can be concluded that for typical aberration values for 3-fold astigmatism and coma after software correction, the shape of the atomic columns can still be modeled by a rounded Gaussian function and therefore, the deviation from the correct scattered intensity is less than $\approx 1 \%$. The effect of 2-fold astigmatism depends on specimen thickness and the magnitude of the aberration. Up to values of $3 \mathrm{~nm}$ for $A_{1}$, deviations from the correct volume are below $\approx 10 \%$ for large number of atoms ( 75 atoms) and less than $\approx 5 \%$ for fewer atoms (less than 25 atoms).

In conclusion, the model-based analysis of HAADF STEM images is shown to be robust for inaccuracies in both cylindrically symmetric and non-cylindrically symmetric aberrations.

\section{Acknowledgments}

The authors acknowledge financial support from the Research Foundation Flanders (FWO, Belgium) through project fundings G.0393.11, G.0064.10, G.0374.13, G.0044.13 and a Ph.D. research grant to A.D.B.

J.V. was supported by funding from the European Research Council under the 7th Framework Program (FP7), ERC Grant No. 246791-COUNTATOMS and ERC Starting Grant No. 278510VORTEX. A.R. thanks the DFG under contract number RO2057/8-1.

The research leading to these results has received funding from the European Union 7th Framework Programme [FP7/2007-2013] under grant agreement no. 312483 (ESTEEM2).

\section{References}

Bals, S., Casavola, M., Van Huis, M., Van Aert, S., Batenburg, K., Van Tendeloo, G., Vanmaekelbergh, D., 2011. Three-dimensional atomic imaging of colloidal core-shell nanocrystals. Nanoletters 11, 3420-3424.

Bals, S., Van Aert, S., Romero, C., Lauwaet, K., Van Bael, M., Schoeters, B., Partoens, B., Yücelen, E., Lievens, P., Van Tendeloo, G., 2012. Atomic scale dynamics of ultrasmall germanium clusters. Nature Communications 3, 897.

Barthel, J., Thust, A., 2010. Aberration measurement in HRTEM: implementation and diagnostic use of numerical procedures for highly precise recognition of diffractogram patterns. Ultramicroscopy 111, 27-46.

Batson, P., 2006. Characterizing probe performance in the aberration corrected stem. Ultramicroscopy 106, 1104-1114.

Born, M., Wolf, E., 1998. Principles of Optics-Electromagnetic Theory of Propagation, Interference and Diffraction of Light, seventh (expanded) ed. Cambridge University Press, Cambridge.

Chen, A., Holt-Hindle, P., 2010. Platinum-based nanostructured materials: synthesis, properties and applications. Chemical Reviews 110, 3767-3804.

De Backer, A., Martinez, G.T., Rosenauer, A., Van Aert, S., 2013. Atom counting in HAADF STEM using a statistical model-based approach: methodology, possibilities, and inherent limitations. Ultramicroscopy 134, 23-33.

den Dekker, A.J., Van Aert, S., van den Bos, A., Van Dyck, D., 2005. Maximum likelihood estimation of structure parameters from high resolution electron microscopy images. Part I: A theoretical framework. Ultramicroscopy 104, 83-106.

Haider, M., Uhlemann, S., Zach, J., 2000. Upper limits for residual aberrations of high-resolution aberration-corrected stem. Ultramicroscopy 81, 163-175.

Ishizuka, K., 2002. A practical approach for STEM image simulation based on the FFT multislice method. Ultramicroscopy 90, 71-83.

Kotaka, Y., 2010. Essential experimental parameters for quantitative structure analysis using spherical aberration-corrected HAADF-STEM. Ultramicroscopy 110 555-562.

Krivanek, O., Corbin, G., Dellby, N., Elston, B., Keyse, R., Murfitt, M., Own, C., Szilagyi, Z., Woodruff, J., 2008. An electron microscope for the aberration-corrected era. Ultramicroscopy $108,179-195$.

LeBeau, J., Findlay, S., Allen, L., Stemmer, S., 2008. Quantitative atomic resolution scanning transmission electron microscopy. Physical Review Letters 100 206101.

LeBeau, J., Findlay, S., Allen, L., Stemmer, S., 2010. Standardless atom counting in scanning transmission electron microscopy. Nanoletters 10, 4405.

Lupini, A., Wang, P., Nellist, P., Kirkland, A., Pennycook, S., 2010. Aberration measurement using the Ronchigram contrast transfer function. Ultramicroscopy 110 891-898.

Martinez, G.T., Rosenauer, A., De Backer, A., Verbeeck, J., Van Aert, S., 2014. Quantitative composition determination at the atomic level using model-based high-angle annular dark field scanning transmission electron microscopy. Ultramicroscopy 137, 12-19.

Nellist, P., 2007. Scanning transmission electron microscopy. In: Hawkes, P.W., Spence, J.C.H. (Eds.), In: Science of Microscopy, vol. I. Springer, New York.

Pennycook, S., Jesson, D., 1991. High-resolution Z-contrast imaging of crystals. Ultramicroscopy 37, 14-38.

Robb, P., Finnie, M., Longo, P., Craven, A., 2012. Experimental evaluation of interfaces using atomic-resolution high angle annular dark field (HAADF) imaging. Ultramicroscopy $114,11-19$

Rosenauer, A., Schowalter, M., 2007. STEMSIM-a new software tool for simulation of STEM HAADF Z-contrast imaging. Springer Proceedings in Physics 120, $169-172$.

Rosenauer, A., Gries, K., Mueller, K., Pretorious, A., Schowalter, M., Avramescu, A., Engl, K., Lutgen, S., 2009. Measurement of specimen thickness and composition in $\mathrm{Al}_{x} \mathrm{Ga}_{1-x} \mathrm{~N} / \mathrm{GaN}$ using high-angle annular dark field images. Ultramicroscopy 109, 1171-1182

Uhlemannm, S., Haider, M., 1998. Residual wave aberrations in the first spherical aberration corrected transmission electron microscope. Ultramicroscopy 72 , 109-119.

Van Aert, S., den Dekker, A.J., van den Bos, A., Van Dyck, D., Chen, J., 2005. Maximum likelihood estimation of structure parameters from high resolution electron microscopy images. Part II: A practical example. Ultramicroscopy 104, 107-125.

Van Aert, S., Verbeeck, J., Erni, R., Bals, S., Luysberg, M., Van Dyck, D., Van Tendeloo, G., 2009. Quantitative atomic resolution mapping using high-angle annular dark field scanning transmission electron microscopy. Ultramicroscopy 109 , 1236-1244.

Van Aert, S., Batenburg, K., Rossel, M., Erni, R., Van Tendeloo, G., 2011. Threedimensional atomic imaging of crystalline nanoparticles. Nature 470, 374-377.

Van Aert, S., Van den Broek, W., Goos, P., Van Dyck, D., 2012. Model-based electron microscopy: from images toward precise numbers for unknown structure parameters. Micron 43, 509-515.

Van Aert, S., De Backer, A., Martinez, G.T., Goris, B., Bals, S., Van Tendeloo, G., Rosenauer, A., 2013. Procedure to count atoms with trustworthy single-atom sensitivity. Physical Review B 87, 064107.

Wong, K., Kirlkland, E., Xu, P., Loane, R., Silcox, J., 1992. Measurement of spherical aberration in stem. Ultramicroscopy 40, 139-150.

Yu, W., Porosoff, M., Chen, J., 2012. Review of Pt-based bimetallic catalysis: from model surfaces to supported catalysts. Chemical Reviews, http://dx.doi.org/10.1021/cr300096b.

Zemlin, F., Weiss, K., Schiske, P., Kunath, W., Herrmann, K., 1978. Coma-free alignment of high resolution electron microscopes with the aid of optical diffractograms. Ultramicroscopy 3, 49-60. 